\title{
A Low Cost Carbon Nanofiber Based Spiral Inductor: Inference and Implementation
}

\author{
John Wiselin, ${ }^{1}$ Sreeja Balakrishnapillai Suseela, ${ }^{2}$ \\ Bycil Viswambaran Jalaja, ${ }^{3}$ Sherin Dhas Sahayadas Padma Ramani, ${ }^{3}$ Rajesh Prasad, \\ Sheeba Devaraj, ${ }^{2}$ Suhaila Shahul, ${ }^{2}$ and Sowmya Swaminathan ${ }^{2}$ \\ ${ }^{1}$ Travancore Engineering College, Oyoor, Kollam District 691516, India \\ ${ }^{2}$ Department of ECE, SSN College of Engineering, Kalavakkam, Chennai 603110, India \\ ${ }^{3}$ PRS College of Engineering and Technology, kerala 695011, India \\ ${ }^{4}$ Satyam College of Engineering and Technology, Aralvaimozhi, Kanyakumari District 629301, India \\ Correspondence should be addressed to Sreeja Balakrishnapillai Suseela; sreejabs@ssn.edu.in
}

Received 2 August 2013; Accepted 28 November 2013; Published 25 February 2014

Academic Editor: Tao Zhang

Copyright (C) 2014 John Wiselin et al. This is an open access article distributed under the Creative Commons Attribution License, which permits unrestricted use, distribution, and reproduction in any medium, provided the original work is properly cited.

\begin{abstract}
This paper investigates the possibilities of using carbon fiber as an inductor material by analyzing its inductive properties. Various shapes such as rectangular, spiral, helical, and cylindrical line structures have been simulated under various constraints using simulation software. Hardware implementations were also tested and both simulation and hardware results show that carbon fibers have the potential to replace copper inductor lines. The implemented spiral inductor produced a quality factor of 40 while producing an inductance of $4 \mathrm{nH}$ at $1.2 \mathrm{GHz}$ frequency.
\end{abstract}

\section{Introduction}

Inductor, a critical passive circuit element, is extensively used in analog signal processing circuits. Inductors designed using copper wires are found to be bulky in nature and also result in significant losses. With the recent advancement in MEMS technology, the size and performance of inductors have become a major factor in analyzing the efficiency of low-power, high frequency circuits. This calls the need for realization of inductors of very small sizes [1-5]. Along with the difficulties of fabricating such small inductors comes the formidable challenge of designing them to electrical specifications. In contrast, carbon fibers are light-weight, corrosion resistant material consisting of fibers about 5 to $10 \mu \mathrm{m}$ in diameter. This analysis queers the disadvantages of copper inductor and provides insights that could potentially open up new applications using carbon fiber.

Inductors are one of the bulk devices which limit the performance in most of the electronic circuits. During the 19th century micro-electro-mechanical inductors played an important role in replacing conventional copper inductors, since the power consumption of the later is higher due to the high current density. As an example the maximum current density of twenty turns of 16 AWG (American Wire Gauge) inductor with $26 \mathrm{~mm}^{2}$ diameter will be about $275 \mathrm{~A} / \mathrm{cm}^{2}$.

Microscale inductors have been investigated by several researchers, and it has been proved that they can be superior to conventional copper inductors [6-10]. However, these investigations have also revealed that phenomena such as the skin effect, proximity effects, eddy current losses, large power consumption, and electric field penetration into the substrate degrade the performance of on-chip inductors at high frequencies. The primary challenge in designing on-chip inductor with high quality factor is reducing the substrate losses and series resistance of inductors [11]. micro-electromechanical (MEM) inductors, with the advanced micromachining techniques, such as Bipolar and CMOS processes, offer high ohmic resistance and confine the substrate losses. On the other hand, to reduce the series resistance, postprocessed $\mathrm{Cu}$ inductors are suggested [12]. To improve the quality factor of the on-chip inductor several approaches have been proposed [13-15]. 
Carbon fibers are strands of conductor embedded in a nonconductive epoxy. This configuration nearly eliminates eddy currents, which otherwise would reduce conductivity limiting it to the surface, thus making it highly conductive at high frequencies. Without eddy currents, the area available for conduction in carbon fiber is much thicker than for a similar amount of copper. The reason for high conductivity of carbon fibers is due to large skin depth. Carbon fibers have ultra high modulus around $1000 \mathrm{GPa}$ and low mass. It offers great flexibility in terms of tuning thermal and mechanical properties through the orientation and number of layups of the fibers. It has many outstanding properties such as high intensity, high modulus, high heat endurance, creep resistance, fatigue endurance, corrosion resistance, and small coefficient of thermal expansion. The internal structure of carbon fiber can be changed by processing under different temperatures so as to change its thermal conduction, thus making it a suitable material for use in aviation and aerospace [16]. Analysis of thermal conductivity of carbon fibers using infrared imaging technology provides a pseudocolour image which describes its temperature distribution. The result of the analysis showed that, initially, there is a rapid increase in the temperature of the material. With time extension, the temperature tends to become mild and gradually stable.

Highly conductive metal materials are the best shielding materials for reflecting and absorbing electromagnetic waves. But they are bulky in nature, difficult to process, expensive and have a tendency to corrode. In contrast, lightweight and anticorrosive properties of polymer materials are extremely suitable for electronic equipment. However, most current polymer materials are not conductive and have no electromagnetic shielding effectiveness. The addition of a conductive filling to polymer material increases the load transfer ability, so that the material can rapidly conduct a load and provide the shielding ability against electromagnetic waves [6]. In addition, the distance of electronic transmission can be shortened, which in turn increases the electromagnetic shielding effectiveness of this kind of composite film prepared by composite filling materials. The electromagnetic shielding effectiveness can appear well within the frequency range of $50 \mathrm{MHz}-1.5 \mathrm{GHz}$.

A design methodology has been developed to analyze the various properties of carbon fiber inductors using existing simulation software-COMSOL Multiphysics and High Frequency Structural Simulator (HFSS). A spiral inductor formed using carbon fiber has been implemented in resonant and amplifier circuits. Practical measurement of inductance was carried out by extracting " $S$ " and " $Y$ " parameters using Network Analyzer. The results show that carbon fibers have the potential to replace conventional inductors.

Inductor and inductive devices formed of a conductive loaded resin-based material is a combination of conductive fibers (nickel plated carbon fiber, silver, or copper) and micron conductive powders (carbon or graphite) [17]. These powders create an additional low level electron exchange when used in combination with the fibers, thus producing further electrical conductivity. The use of such inductors reduces the cost of manufacturing process. These inductors can be molded into infinite shapes using conventional
TABLE 1: Comparison of properties between copper and carbon fiber.

\begin{tabular}{lcc}
\hline Properties & Copper & $\begin{array}{c}\text { Carbon } \\
\text { fiber }\end{array}$ \\
\hline Density $\left(\mathrm{Kg} / \mathrm{m}^{3}\right)$ & 8700 & 1780 \\
Electrical conductivity $\left(\mathrm{Kg} / \mathrm{m}^{3}\right)$ & $5.998 e 7$ & $8.5 e 7$ \\
Coefficient of thermal expansion $(1 / \mathrm{K})$ & $17 e-6$ & $2.15 e-6$ \\
Heat capacitance at constant pressure $(\mathrm{J} / \mathrm{kg} \cdot \mathrm{K})$ & 385 & 710 \\
Electron mobility $\left(\mathrm{m}^{2} / \mathrm{V} \cdot \mathrm{s}\right)$ & 0.003 & 1.5 \\
Thermal conductivity $(\mathrm{W} / \mathrm{m} \cdot \mathrm{K})$ & 400 & 1000 \\
Poisson's ratio & 0.35 & 0.77 \\
Young's modulus $(\mathrm{Pa})$ & $110 e 9$ & $220 e 9$ \\
Resistivity $(\Omega \mathrm{m})$ & $1.72 e-8$ & $1.45 e-8$ \\
\hline
\end{tabular}

forming methods such as injection molding or extrusion process and exhibit excellent thermal dissipation characteristics. The molded fibers provide resistivity ranging between 5 and $25 \mathrm{ohms}$ per square. Resistivity of the inductors can be varied by varying the doping parameters or by selecting a suitable resin.

The study of properties of carbon fiber reinforces that inductor design using carbon fiber is found to be more efficient than copper. This can be inferred from the properties listed in Table 1.

\section{Analysis and Inference of Carbon Fiber Inductor}

2.1. Small Signal Analysis of Carbon Fiber Inductor. The small signal analysis of an inductor is based on the principle that if an inductor's magnetic material is nonlinear, then the inductance depends upon the current passing through it [16]. The simulation is performed on a model inductor with a nonlinear magnetic core that shows a changing inductance when the current increases. The small signal inductance has been investigated as a function of current through the inductor using the structure shown in Figure 1.

The module used is $2 \mathrm{D}$ axisymmetric space dimensionAC/DC module. The geometry consists of a magnetic iron core with carbon fibers wound over it. The operating frequency is set at $10 \mathrm{kHz}$.

The small signal inductance is plotted versus the DC bias current and is shown in Figure 2. For low DC voltages the inductance is maximum $(23 \mathrm{mH})$, and when the voltage increases the inductance of the proposed test geometry decreases to $11 \mathrm{mH}$. The inductance drops to zero as a consequence of the saturation of the core. The saturation can be visualized by plotting the relative permeability of the iron core (Figure 3).

2.1.1. Mutual Inductance. The mutual inductance between a primary and secondary single turn carbon fiber coil in a concentric coplanar arrangement is computed using a DC (steady-state) model [2]. The induced current in the secondary coil is computed using an AC (frequency-domain) 


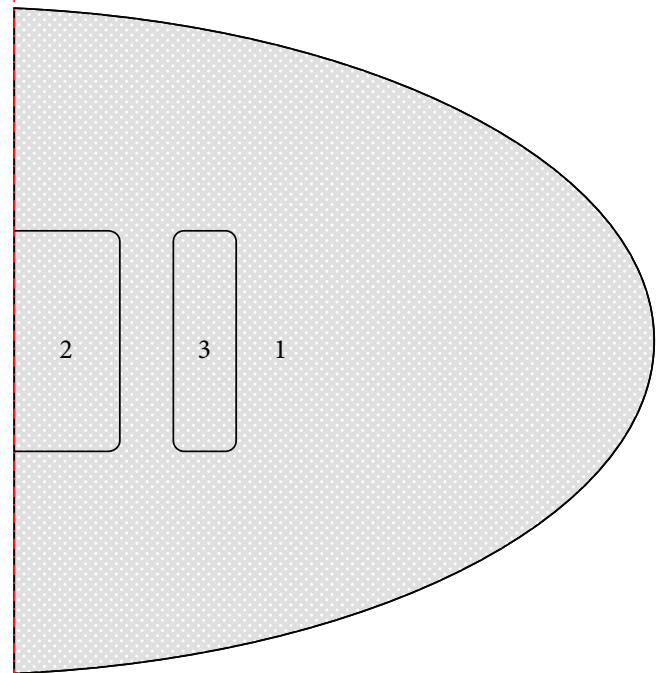

FIGURE 1: Geometry profile of the inductor which is used to study the small signal analysis where the domain radius is $3 \mathrm{~cm}$, inductor length is $2 \mathrm{~cm}$, core radius is $5 \mathrm{~mm}$, coil outer radius is $10.5 \mathrm{~mm}$, and the coil inner radius is $7.5 \mathrm{~mm} ; 1$ represents the air domain; 2 and 3 represent the magnetic core and thickness of the coil, respectively.

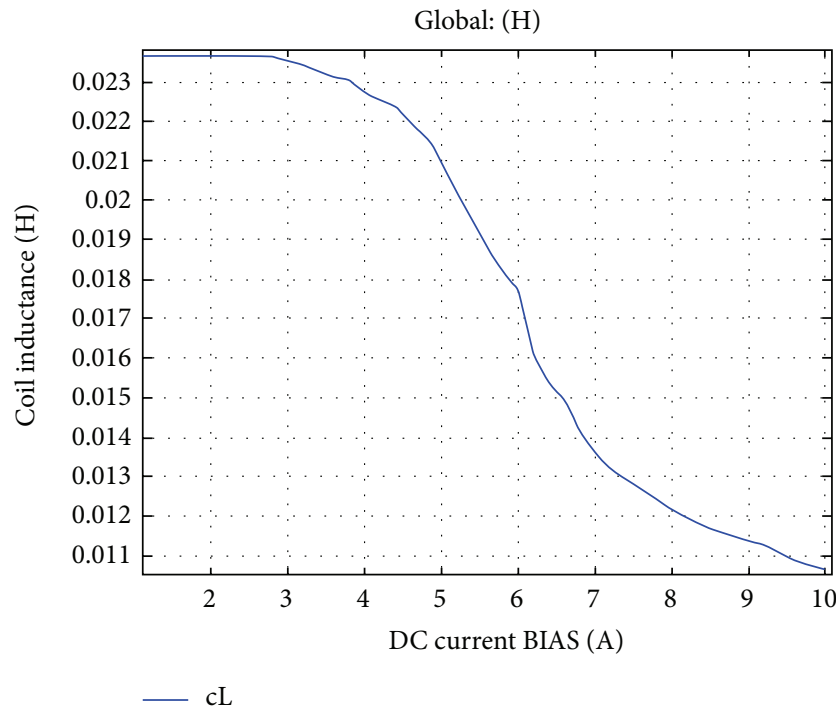

FIGURE 2: Plot of DC current bias versus coil inductance.

analysis. The relationship between the AC induced currents and the DC inductance has been discussed in the following sections.

A current of 1 Ampere flows through a single turn coil of radius $R_{1}=100 \mathrm{~mm}$ (Figure 4 ). A secondary coil, $R_{2}=$ $10 \mathrm{~mm}$, is concentric with the primary and in the same plane. The coils are modeled in the $2 \mathrm{D}$ axisymmetric space dimension.

In the limit as $R_{1} \gg R_{2}$, the analytic expression for the mutual inductance between the two coils is

$$
M=\frac{\pi \mu_{0} R_{2}^{2}}{2 R_{1}},
$$

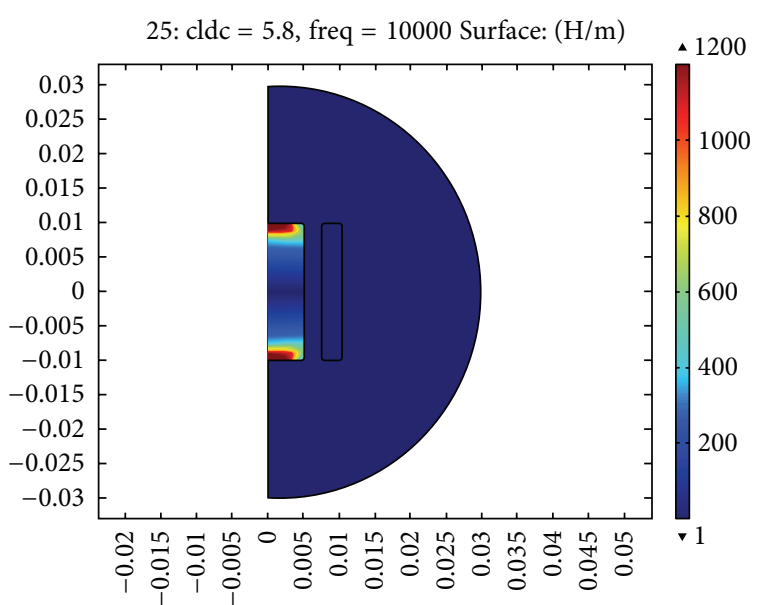

FIGURE 3: Relative permeability distribution.

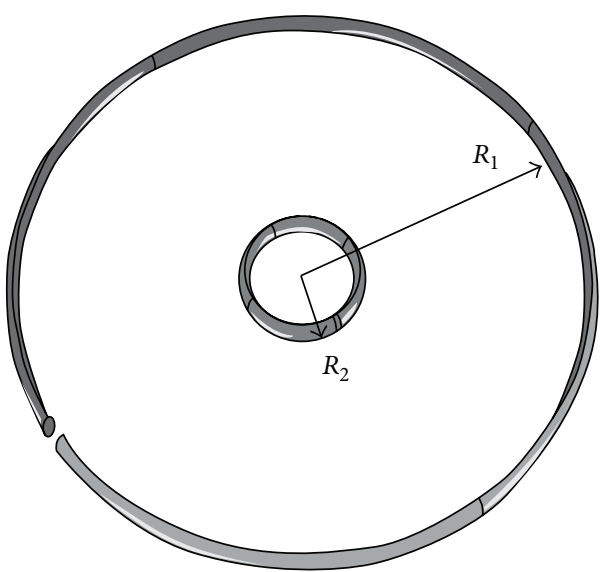

FIgURE 4: Concentric coplanar arrangement.

where $M$ is mutual inductance, $\mu_{0}$ is permeability in free space, $R_{1}$ is radius of a primary coil, and $R_{2}$ is radius of a secondary coil.

The two concentric coils are modeled in a 2D axisymmetric sense, as shown schematically in Figure 5.

2.1.2. DC Analysis. The modeling domain is surrounded by a region of infinite elements, which is a way to truncate a domain which stretches to infinity. Although the thickness of the infinite element domain is finite, it can be thought of as a domain of infinite extent. The coils are both modeled using the Single Turn Coil Domain feature, which can be thought of as introducing an infinitesimal slit in an otherwise continuous torus. The Single Turn Coil feature can be used to excite the coil, to represent the open circuit case and the closed torus case, and to model an external load. The primary coil is excited by specifying $1 \mathrm{~A}$ of current.

The DC magnetic flux is plotted in Figure 6. The mutual inductance is evaluated using the line integral approach of the magnetic flux, as well as the point integral of the magnetic vector potential. The value of $M$ is found to be $1.978 \mathrm{nH}$. 


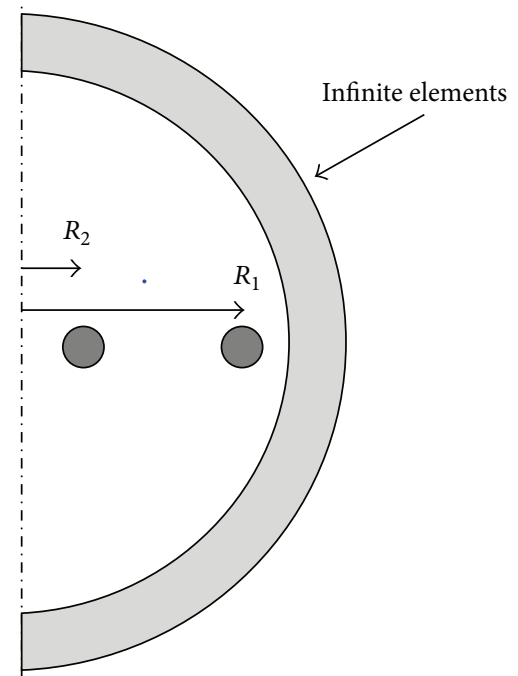

FIGURE 5: A schematic representation of the 2D axisymmetric model of the concentric coils.

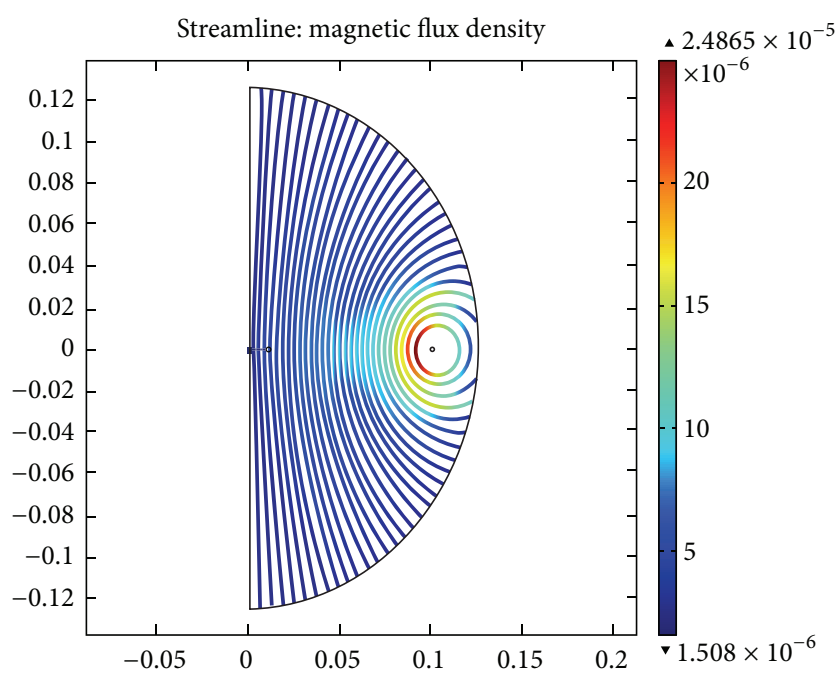

FIgURe 6: Magnetic flux density distribution.

2.1.3. AC Analysis. For the AC case, a $1 \mathrm{kHz}$ sinusoidal timevarying current drives the primary coil. This can either induce currents in the secondary coil or induce a voltage difference if the coil is modeled as an open circuit. The secondary coil domain uses the Single Turn Coil Domain to model both the open circuit and the closed circuit case.

2.1.4. Open Circuit Case. To model the open circuit case, the current through the coil is specified to be $0 \mathrm{~A}$, which specifies that there is no current flowing through the coil. The Single Turn Coil Domain feature will introduce a coil voltage that causes no current to flow. The average of the induced currents over the cross section is zero; that is, there is no net current flow through the coil. Since the Single Turn Coil Domain computes the loop potential, this voltage induced across

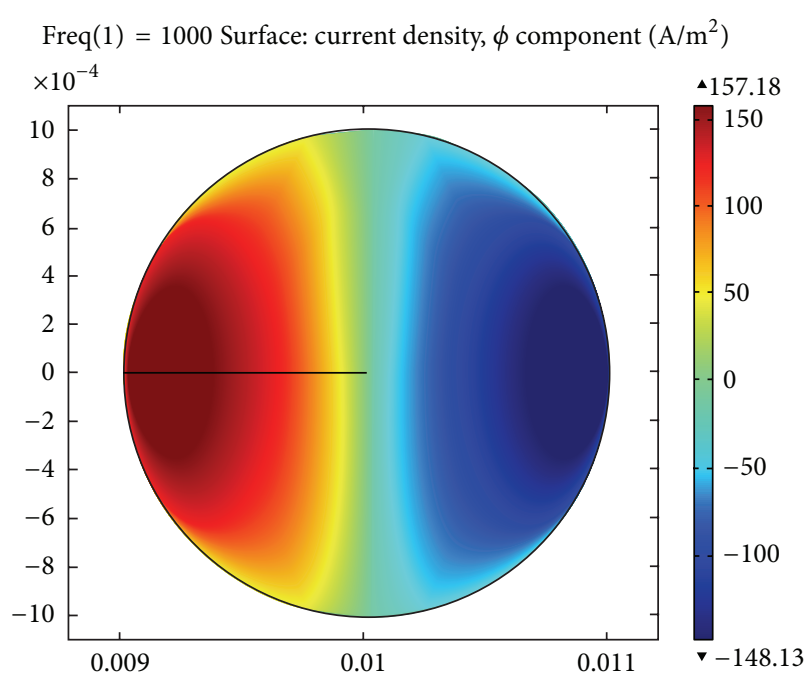

FIGURE 7: Induced currents in the coil for open circuit case.

the coil can be used to compute the mutual inductance which is given as follows:

$$
M=\frac{V}{i \omega I},
$$

where $V$ is coil voltage, $I$ is induced current, $\omega$ is frequency of the coil, and $I$ is current flow in the coil.

The computed mutual inductance is found to be 1.973 $0.004 \mathrm{inH}$. The small imaginary component is due to the capacitive effects. Figure 7 shows the induced current flow in the coils during open circuit case.

2.1.5. Closed Circuit Case. On the other hand, to model the closed circuit case, the voltage drop across the coil is fixed at $0 \mathrm{~V}$. Although this seems to imply a short circuit, the reactance of the copper coil is inherently included, so the case being modeled is analogous to a closed continuous loop of wire.

The skin effect is clearly visible; the current is being driven to the boundaries of the domain. The total induced current around the secondary coil is $-0.01675-0.02677 i$; the imaginary component implies a reactive current. Figure 8 shows the induced current flow in the coils during open circuit case.

2.1.6. Calculating the Inductance of a Spiral Coil. In this analysis, COMSOL Multiphysics is used to solve the magnetic fields surrounding the coil that is placed in electrical circuit and calculate the inductance of the coil. In some cases the analytic approach could be difficult and with increasing complexity of geometries of some coils these are sometimes quite difficult to calculate [4]. The use of finite element method can be a good alterative of solving such problems. The aim of the research is to calculate the inductance of the coil.

2.1.7. Coil Parameters. Number of turns is $N=9$; outer diameter is $d_{\text {out }}=142 \mathrm{~mm}$; inner diameter is $d_{\text {in }}=30 \mathrm{~mm}$; 


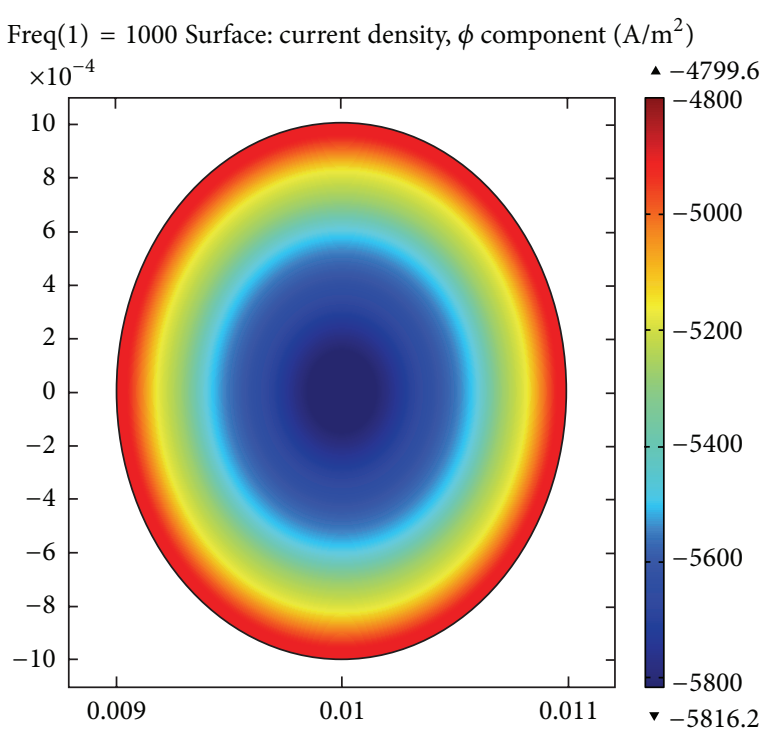

Figure 8: Induced currents in the coil for the closed circuit case.

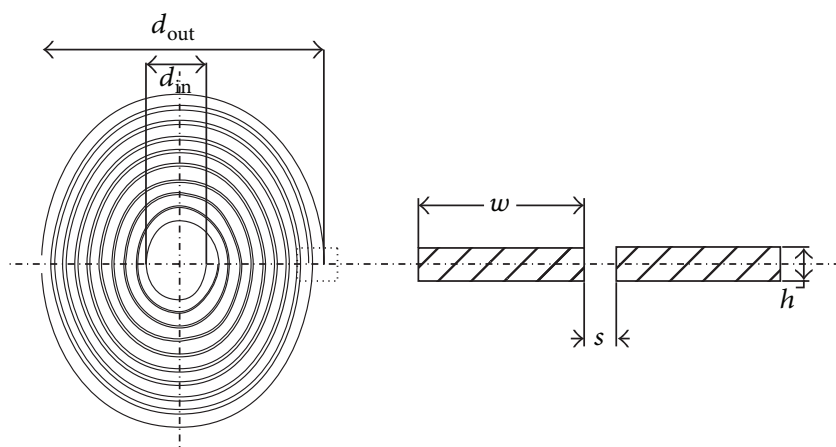

FIGURE 9: Spiral coil topology.

spacing of turns is $s=1 \mathrm{~mm}$; section of wire is $w \times h=$ $5 \times 0.8 \mathrm{~mm}$.

2.1.8. Coil Model. Geometry of this study is created in $2 \mathrm{D}$ axisymmetric space dimension-AC/DC module. For simplicity and initial testing a circular spiral coil is considered. Figures 9 and 10 show topology and the spiral coil models, respectively.

Because the coil is created in $2 \mathrm{D}$ axisymmetric space dimension, the model is simplified and the coil is created with nine circles. Geometry consists of 10 domains, one air domain surrounding the coil and the remaining are coil domains.

The coil material is selected as carbon fiber with conductivity $\sigma=8.5 e 7 \mathrm{~S} / \mathrm{m}$. Coil is driven by DC current $I_{0}=$ $20 \mathrm{~A}$. Inductance of the coil is calculated from total magnetic energy created by current passing through the coil using the following formula:

$$
W_{m}=\frac{1}{2} L I_{0}^{2}
$$

where $W_{m}$ is total magnetic energy, $L$ is inductance of coil, and $I_{0}$ is DC current.

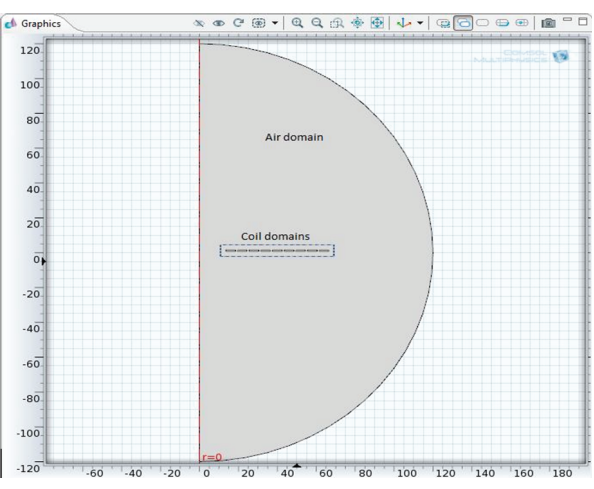

(a)

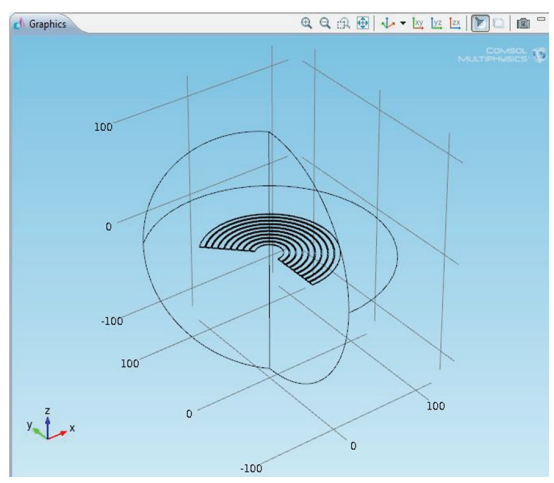

(b)

FIGURE 10: Simplified model of coil, (a) 2D and (b) 3D representation.

2.1.9. Simulation Results. Total magnetic energy $W_{m}$ is $10.534 e-4 \mathrm{~J}$; inductance of coil $L$ is $5.26 \mu \mathrm{H}$.

Figure 11 shows the magnetic flux density distribution of the spiral coil and the magnetic field is strongest in the middle of the coil and decreases with increasing radius of coil. Figure 12 shows the magnetic flux density contour, which clearly shows the strongest field in the middle of the coil. After this small signal analysis it is confirmed by the author that the carbon fiber material could be used for future fabrication of inductor.

\section{Rectangular Spiral Inductor Model Analysis}

For the final implementation a rectangular spiral inductor has been selected since it is a well studied topology and most of the on-chip applications use the rectangular spiral inductor. As a first step a spiral inductor model has been considered for analysis using HFSS in order to determine the value of inductance and $Q$ factor.

A spiral carbon fiber coil of 2.5 turns is modeled using HFSS with thickness of $2 \mu \mathrm{m}$ and metal width of $4 \mu \mathrm{m}$ as shown in Figure 13. The inductor is located on an oxide layer with thickness of $8 \mu \mathrm{m}$ on a $10 \Omega \mathrm{cm}$ silicon wafer of thickness $200 \mu \mathrm{m}$. The conductivity of carbon fiber is $8.5 \times 10^{7}$ Siemens $/ \mathrm{m}$. Rectangular wave ports have also been assigned during the simulation. Radiation boundary is applied as shown in Figure 14 to the spiral inductor. 


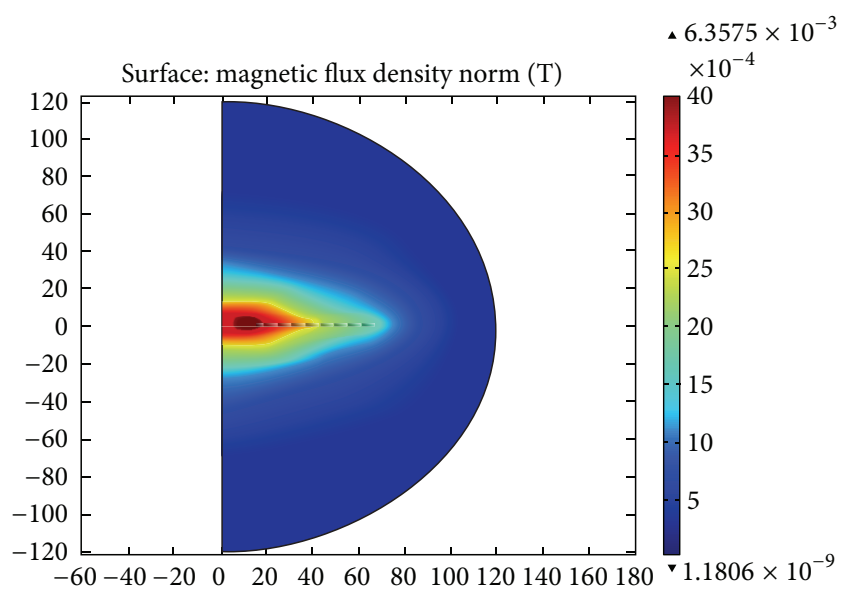

FIGURE 11: Magnetic flux density—surface.
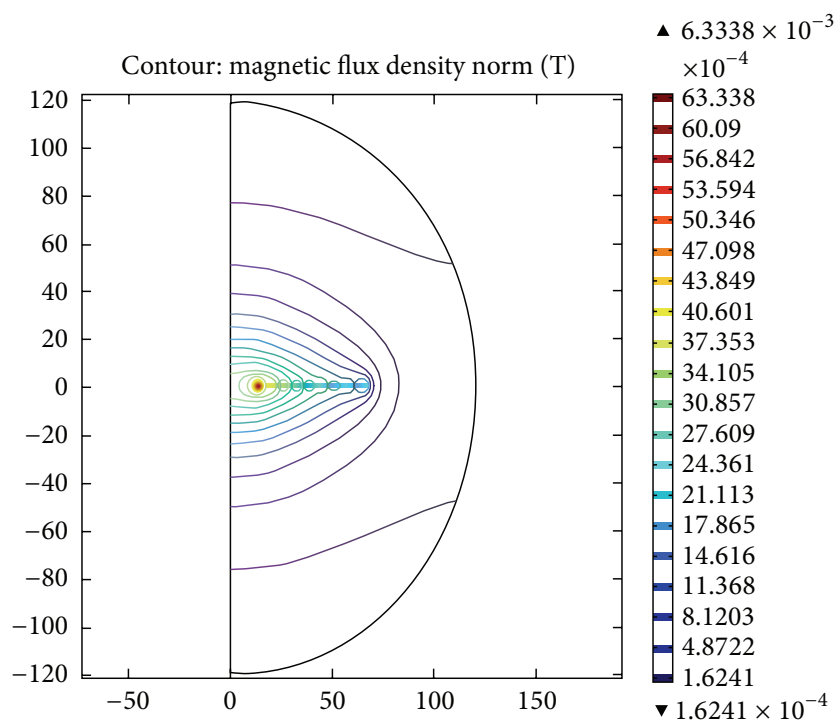

Figure 12: Magnetic flux density—contour.

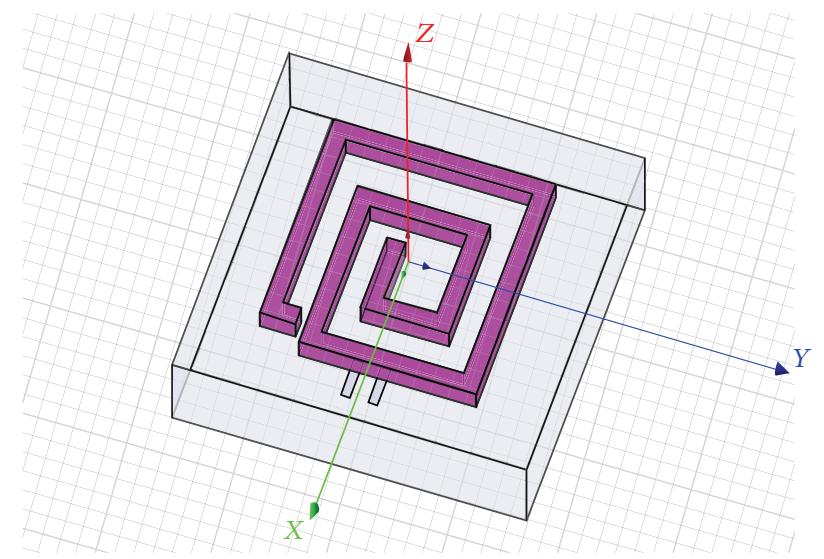

FIGURE 13: Model illustrating spiral coil embedded on a silicon base.

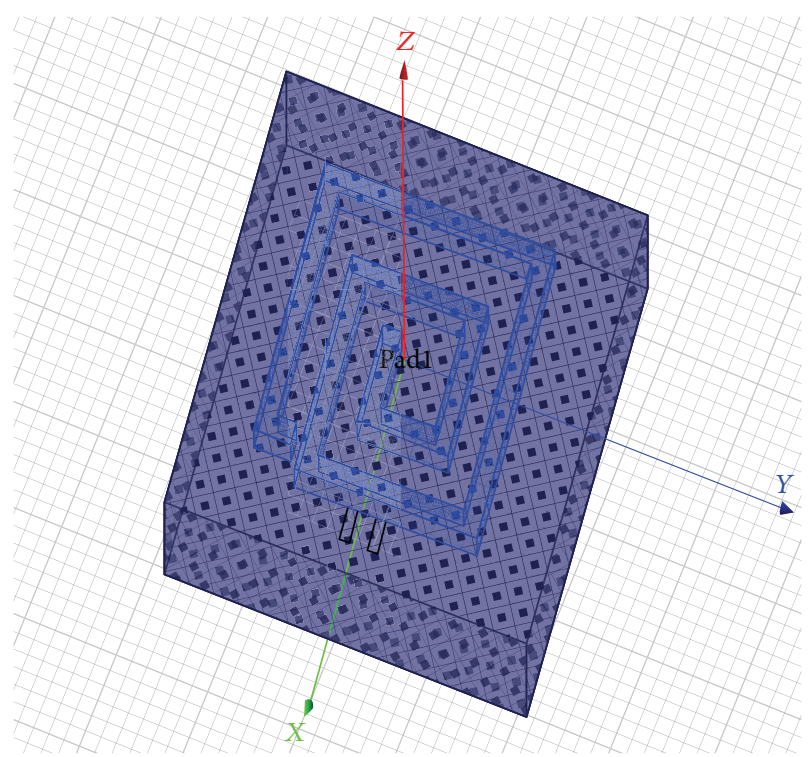

FIGURE 14: Inductor model with radiation boundary.

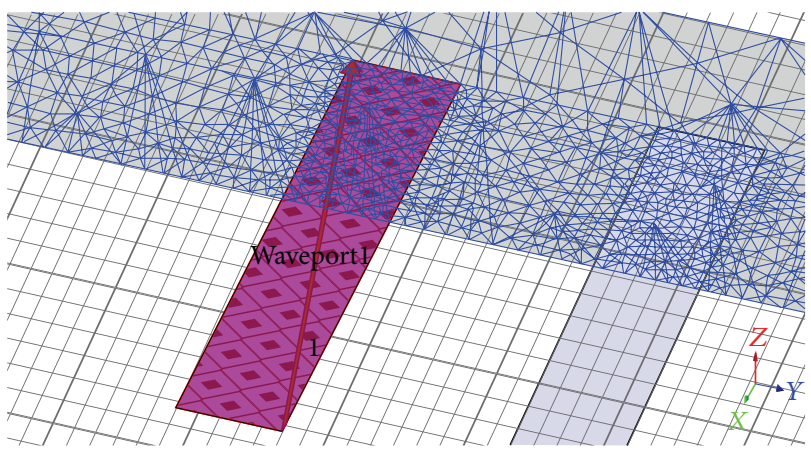

FIGURE 15: Model illustrating wave port excitation.

The excitation is assigned as wave port for the inductor model as shown in Figures 15 and 16.

The simulation of the spiral inductor model has been carried out in the frequency range of $1-5 \mathrm{GHz}$. The sweep type is chosen as discreet and the frequency sweep type that is selected is linear step.

3.1. Simulation Results. The quality factor of the inductor is simulated with respect to the frequency and is plotted in Figure 17. The range of $Q$ lies between 24.10 and 24.45 for the frequency range of $1-5 \mathrm{GHz}$. The simulated magnitude of impedance lies between 950 and 600 over a frequency range of 1 to $10 \mathrm{GHz}$. However the quality factor and the impedance fall at high frequencies; comparing with copper material both these factors are improved to a certain extent. The range of inductance is found to lie between 0.11097 and 0.11135 .

\section{Spiral Inductor Fabrication}

The proposed model is based on using the silicon wafer as an on-wafer test-fixture with the carbon fiber embedded on 


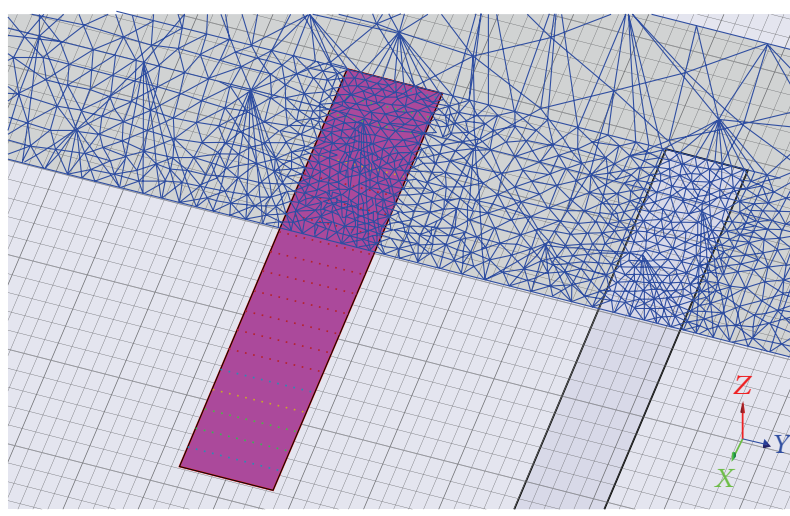

FIGURE 16: Wave port with mode lines.

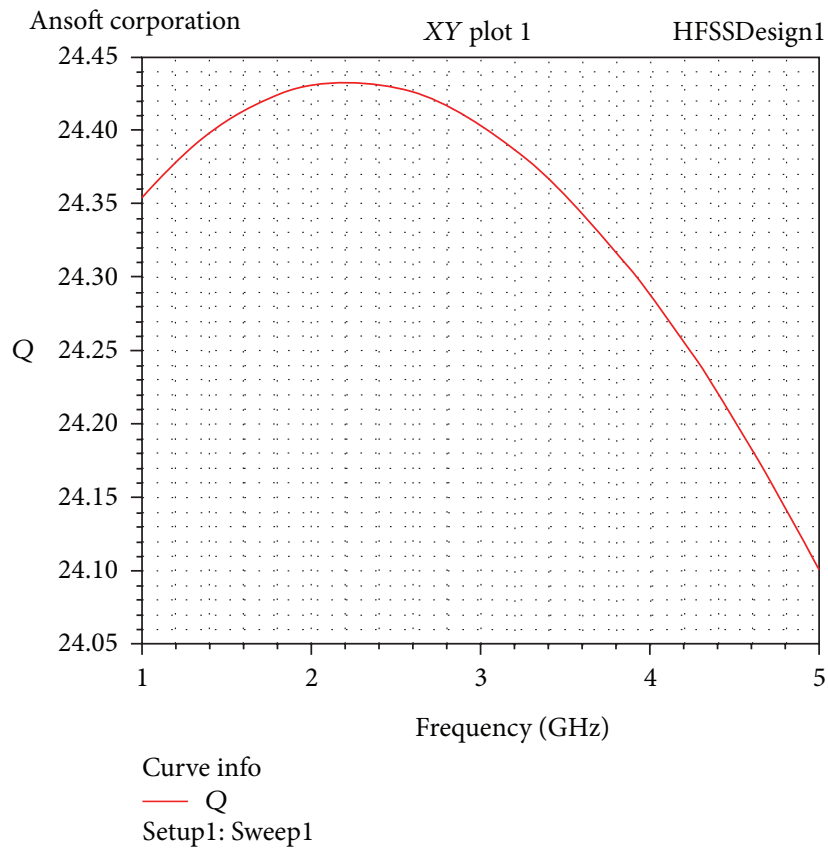

FIGURE 17: Plot of Quality Factor versus frequency.

it using the adhesive silver paste. Silver paste is a conductive solder paste which has excellent resistivity, good soldering ability and adhesion strength. The paste has good adhesion to glass, plastics, and dielectrics. The spherical ultra fine silver powder is readily dispersible in multiple base formulations. Inductor model has been designed by using silver paste as an adhesive to implant the fiber on the wafer. But the binding of the fiber on the base material was not strong enough which ruled out the possibility of using silver paste to design the inductor. The authors have used silver paste to make the fabrication as cost effective. The intention of this research was to test whether the fiber material could be used for inductors. The nickel coated flexible fibre bundle with the diameter $0.15 \mathrm{~mm}$ has been used in this fabrication where the diameter of each strand was $150 \mathrm{um}$. The fabricated low cost inductor is shown in Figure 18.

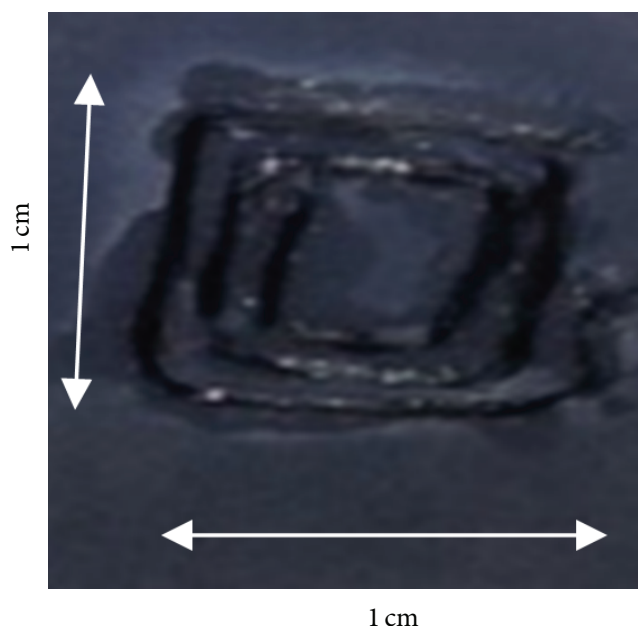

FIGURE 18: Image of implemented low cost carbon fiber inductor.

\section{Measurements and Testing}

The $S$-parameters were measured with the help of an HP8510C network analyzer and a corresponding cascade Microtech ground-signal-ground probing station in the frequency range of $0.1 \mathrm{GHz}$ to $1.5 \mathrm{GHz}$. Dummy pads were designed to extract the parameters of the designed inductor. The parasitic parallel capacitance and the series contact resistance between the substrate and the contact pads of the inductor are deembedded via the dummy patterns and the measured $S$-parameters are then transformed to $Y$ parameters from which the inductance and the quality factors are calculated using the following equation and this is shown in Table 2:

$$
\begin{gathered}
Q=\frac{-\operatorname{Im} a g\left(1 / Y_{11}\right)}{\operatorname{Re} \operatorname{al}\left(1 / Y_{11}\right)}, \\
L=\frac{\operatorname{Im} \operatorname{ag}\left(1 / Y_{11}\right)}{2 \pi f}
\end{gathered}
$$

\section{Conclusion}

In this paper we proposed a low cost carbon fiber inductor. The spiral inductor designed by embedding the carbon fiber on a silicon wafer has been fabricated and tested. The device is fabricated with ultra low cost since carbon fiber material is much cheaper. In addition to this a low cost fabrication is also employed in this work. The fabricated device produces a maximum $Q$ factor of 40.5 at $1.2 \mathrm{GHz}$. The self-resonant frequencies are above $10 \mathrm{GHz}$, which are above the range of the network analyzer used. The self-resonant frequencies are predicted to be in the range of $20 \mathrm{GHz}-30 \mathrm{GHz}$, and with these self-resonant frequencies the proposed inductor can be used for high frequency applications since the energy loss is low. Further, the analysis in extracting the equivalent circuit parameters is expected. Also with further improvements in the fabrication the inductance could be increased at high 
TABLE 2: Inductance and quality factor measurements of low cost inductor.

\begin{tabular}{lcc}
\hline Frequency $(\mathrm{GHz})$ & Inductance $L(\mathrm{nH})$ & $Q$ \\
\hline 0.544 & 10.153 & 30.0 \\
0.630 & 12.947 & 31.9 \\
0.700 & 9.452 & 32.3 \\
0.772 & 5.249 & 30.4 \\
0.945 & 4.290 & 29.5 \\
1.000 & 3.328 & 26.8 \\
1.100 & 3.437 & 36.0 \\
1.200 & 2.271 & 40.5 \\
1.350 & 4.189 & 35.9 \\
1.400 & 5.310 & 36.3 \\
1.500 & 5.224 & 3.5 \\
\hline
\end{tabular}

frequencies above $5 \mathrm{GHz}$. Better fabrications will definitely improve the characteristics of the inductor.

\section{Conflict of Interests}

The authors declare that there is no conflict of interests regarding the publication of this paper.

\section{References}

[1] A. P. Boutz and W. B. Kuhn, "Performance of high-Q inductors in LTCC using FTTF techniques," IEEE Transactions on Components, Packaging and Manufacturing Technology, vol. 1, no. 8, pp. 1125-1131, 2011.

[2] I. J. Bahl, "High-performance inductors," IEEE Transactions on Microwave Theory and Techniques, vol. 49, pp. 654-664, 2001.

[3] C. J. Chao, "Characterization and modeling of on-chip spiral inductors for Si RF ICs," IEEE Transactions on Semiconductor Manufacturing, vol. 15, pp. 19-29, 2002.

[4] D.-M. Fang, X.-H. Li, Q. Yuan, and H.-X. Zhang, "Design, simulation, and characterization of variable inductor with electrostatic actuation fabricated by using surface micromachining technology," IEEE Transactions on Electron Devices, vol. 57, no. 10, pp. 2751-2755, 2010.

[5] H. Chang, Y.-M. Yen, and K.-D. Huang, "Electromagnetic shielding by composite films prepared with carbon fiber, $\mathrm{Ni}$ nanoparticles, and multi-walled carbon nanotubes in polyurethane," Materials Transactions, vol. 51, no. 6, pp. 1145-1149, 2010.

[6] H. Sugawara, Y. Yoshihara, H. Ito, K. Okada, and K. Masu, "Wide-range RF variable inductor on Si CMOS chip with MEMS actuator," in Proceedings of the 34th European Microwave Conference, pp. 701-704, October 2004.

[7] J. Salvia, J. A. Bain, and C. P. Yue, "Tunable on-chip inductors up to $5 \mathrm{GHz}$ using patterned permalloy laminations," in Proceedings of the IEEE International Electron Devices Meeting (IEDM '05), pp. 943-946, December 2005.

[8] I. Zine-El-Abidine, M. Okoniewski, and J. G. McRory, "RF MEMS tunable inductor using bimorph microactuators," in Proceedings of the International Conference on MEMS, NANO and Smart Systems (ICMENS '05), pp. 436-437, July 2006.
[9] C. Patrick Yue and S. Simon Wong, "Physical modeling of spiral inductors on silicon," IEEE Transactions on Electron Devices, vol. 47, no. 3, pp. 560-568, 2000.

[10] J. Cranickx and M. Steyaert, Wireless CMOS Frequency Synthesizer Design, Kluwer Academic Publishers, Norwell, Mass, USA, 1998.

[11] H.-H. Hsieh and L.-H. Lu, "A high-performance CMOS voltagecontrolled oscillator for ultra-low-voltage operations," IEEE Transactions on Microwave Theory and Techniques, vol. 55, no. 3, pp. 467-473, 2007.

[12] H.-C. Chen, C.-H. Chien, H.-W. Chiu et al., "A low-power low-phase-noise LC VCO with MEMS cu inductors," IEEE Microwave and Wireless Components Letters, vol. 15, no. 6, pp. 434-436, 2005.

[13] E.-C. Park, Y.-S. Choi, J.-B. Yoon, S. Hong, and E. Yoon, "Fully integrated low phase-noise VCOs with on-chip MEMS inductors," IEEE Transactions on Microwave Theory and Techniques, vol. 51, no. 1, pp. 289-296, 2003.

[14] P. Park, C. S. Kim, M. Y. Park, S. D. Kim, and H. K. Yu, "Variable inductance multilayer inductor with MOSFET switch control," IEEE Electron Device Letters, vol. 25, no. 3, pp. 144-146, 2004.

[15] S. Zhou, X.-Q. Sun, and W. N. Carr, "Micro variable inductor chip using MEMS relays," in Proceedings of the International Conference on Solid-State Sensors and Actuators, vol. 2, pp. 11371140, June 1997.

[16] H. Li and K. Banerjee, "High-frequency analysis of Carbon Nanotube interconnects and implications for on-chip inductor design," IEEE Transactions on Electron Devices, vol. 56, no. 10, pp. 2202-2214, 2009.

[17] H. Jiang, Y. Wang, J.-L. A. Yeh, and N. C. Tien, "On-chip spiral inductors suspended over deep copper-lined cavities," IEEE Transactions on Microwave Theory and Techniques, vol. 48, no. 12, pp. 2415-2423, 2000. 

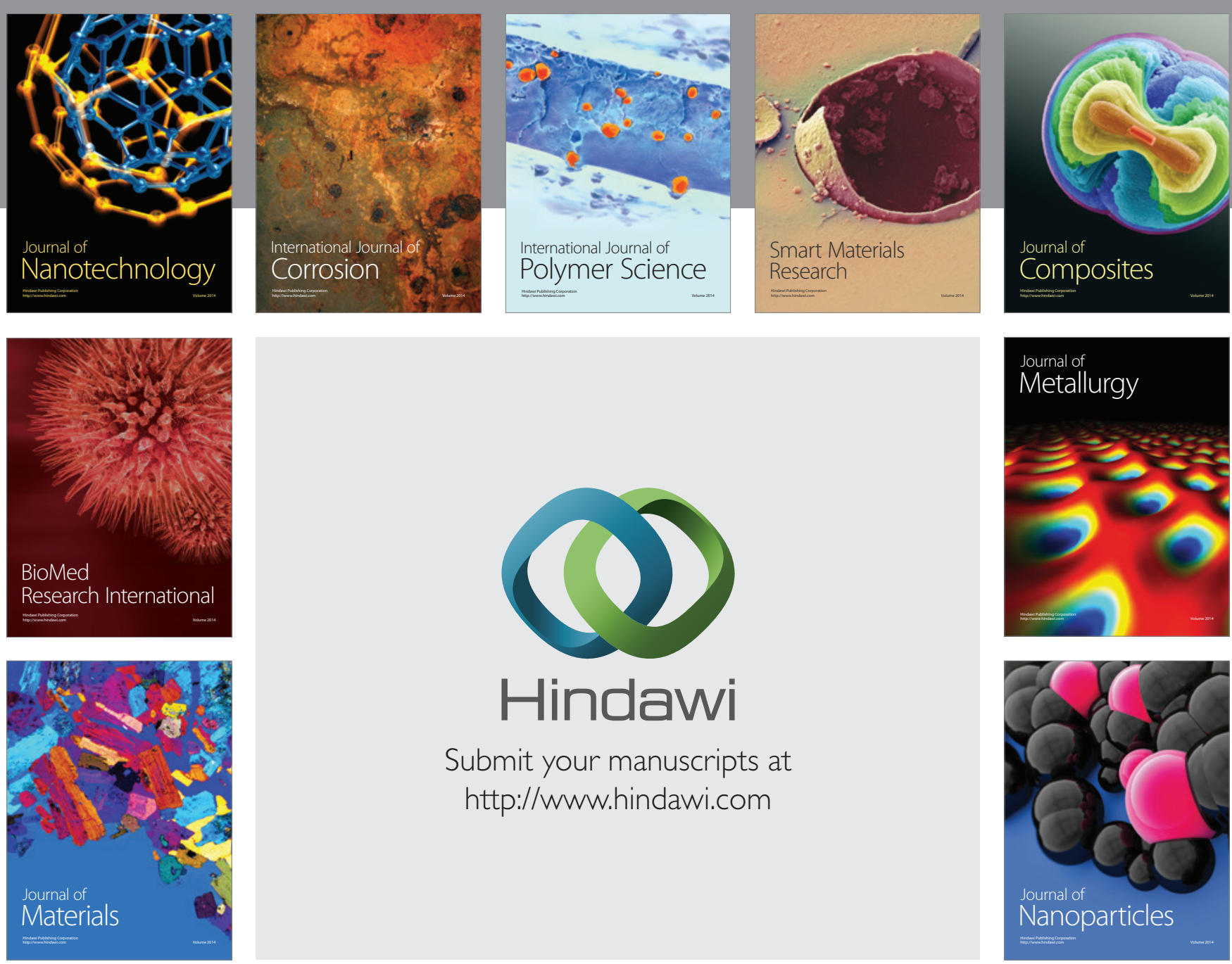

Submit your manuscripts at http://www.hindawi.com
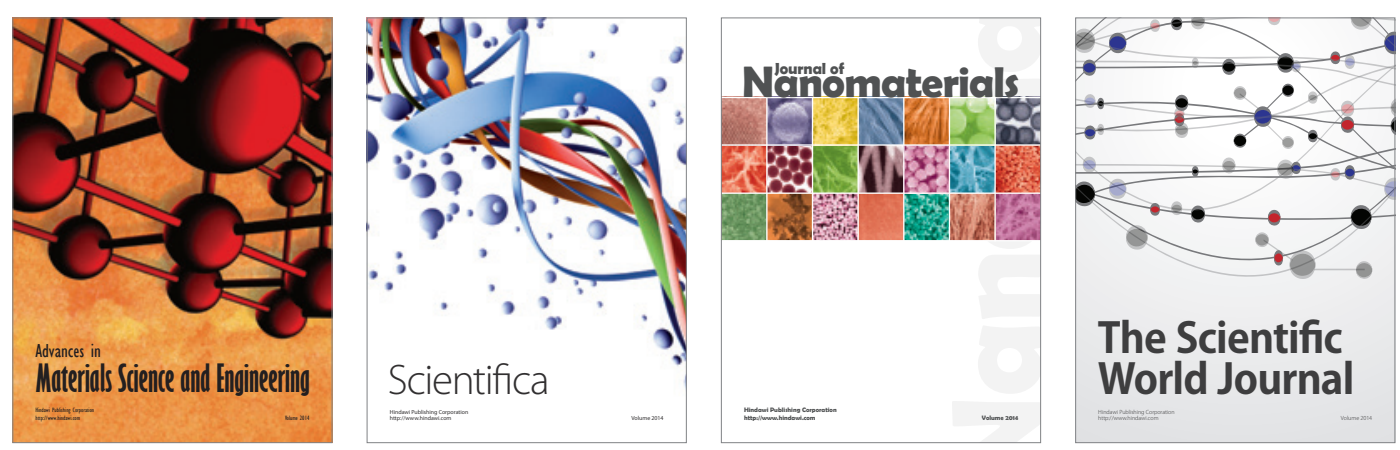

\section{The Scientific World Journal}
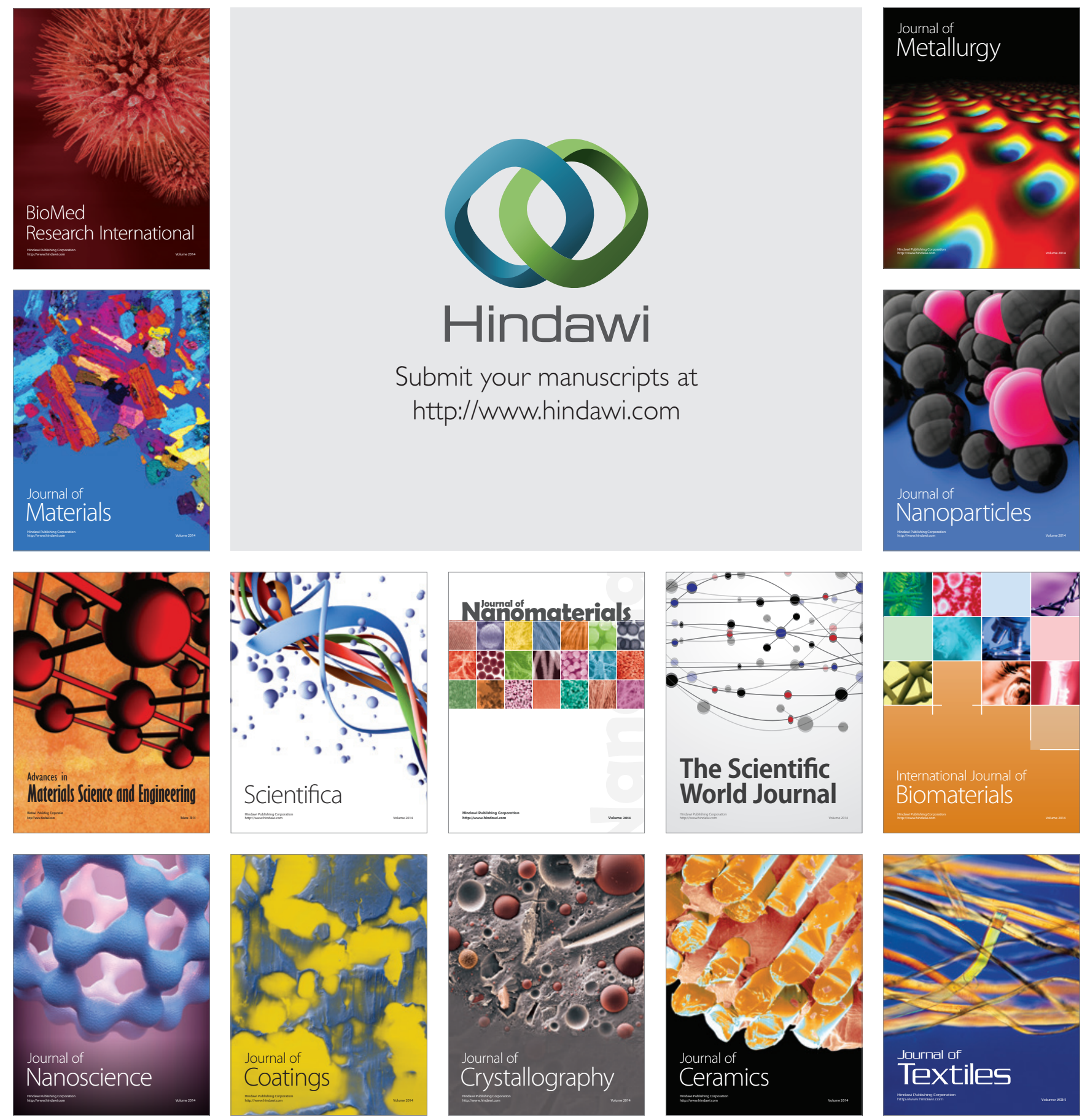